\title{
A "SOFT" SUPERPARAMAGNETIC STATE AND BOUNDARIES OF ITS STABILITY IN AN ISING MODEL OF A DISORDERED GRANULAR MAGNET
}

\author{
E. E. Kokorina, M. V. Medvedev \\ Institute of Electrophysics, the Urals Division, Russian Academy of Sciences \\ 106 Amundsen Str., Ekaterinburg, 620016, Russia
}

(Received September 5, 2001)

\begin{abstract}
An Ising model of a disordered granular magnet is proposed. In this model, identical grains with approximately $N \approx 10^{4}-10^{5}$ magnetic atoms are placed at random into a nonmagnetic matrix, Ising spins inside each grain are coupled with each other by a strong ferromagnetic exchange, and the spins of different granules coupled by a weak random exchange interaction which is described by a nonsymmetric Gaussian distribution.

The model exhibits two critical temperatures of magnetic ordering: first, local ferromagnetic ordering appears inside grains in the absence of a long-range correlation between spontaneous magnetic moments of different grains; then, at a lower temperature either a long-range superferromagnetic state or a macrospin glass one is established over the whole sample. Above the upper critical temperature the usual paramagnetic behaviour of Ising spins is observed, and a "soft" superparamagnetic state (with temperature-dependent absolute values of spontaneous magnetic moments of grains) is established between the upper and lower critical temperatures.

The partition function of the model is calculated in the molecular-field approximation, and the temperature dependence of the linear and nonlinear magnetic susceptibilities in the "soft" superparamagnetic state is analyzed. As well the de Almeida-Thouless instability line at finite magnetic fields is calculated for the case when the system shows a transition from the superparamagnetic state into a low-temperature macrospin glass one.
\end{abstract}

Key words: granular magnet, Ising model, magnetic ordering, disordered system

PACS number(s): 64.60.Cn, 05.50.+q, 75.10.Nr

\section{INTRODUCTION}

Recently, the authors of this paper have considered an Ising model of a granule ferromagnet $[1,2]$ in which mesoscopic precipitates of magnetic atoms of identical sizes (granules) form a regular lattice inside a nonmagnetic matrix. The magnetic moments of the atoms inside each granule are coupled by a strong ferromagnetic interaction between the nearest neighbors, and the magnetic moments of the neighboring granules are coupled by a weak long-range ferromagnetic interaction. In such a model there exist two critical temperatures of magnetic ordering: an upper critical temperature $T_{c 1}$ of magnetic ordering, corresponding to a local ordering, when the spins become ordered inside a granule, but the directions of the total magnetic moments of different granules do not correlate with one another, and a lower critical temperature $T_{c 2}$, corresponding to the global superferromagnetic order, when the total spontaneous magnetic moments of different granules prove to be aligned. It was assumed that the magnetic granules in a nonmagnetic matrix have the same size and shape and a regular distribution as atoms in a regular crystal lattice.

In the real granular magnets the precipitates of magnetic atoms are distributed, as a rule, in a random way and because of that intergranular interactions are also random. There are two main reasons for investigating the model of such a magnet. On the one hand, the magnetic susceptibility of $\mathrm{Cu}-\mathrm{Co}$ supersaturated solid solu- tions with precipitation of cobalt granules was found to have maxima [3] in the temperature range of $100-500 \mathrm{~K}$, depending on the composition of the alloy and the time of its holding at a temperature. This phenomenon was interpreted as the appearance of a macrospin glass. In connection with that it is interesting to reveal, what peculiarities in magnetic characteristics of granule system can be caused by the disordered effects in intergranular interactions.

On the other hand, the Ising model of disordered granule magnet with Gaussian random intergranular exchange interactions let us make comparison with well known Sherrington-Kirkpatrick model of spin glass [4]. This help us to understand, what is the difference in the behaviour between usual spin glass of individual magnetic moments and macrospin glass of mesoscopic supermagnetic granule moments.

\section{AN ISING HAMILTONIAN AND THE PARTITION FUNCTION FOR GRANULE MAGNET IN THE MOLECULAR FIELD APPROXIMATION}

Let the granules of magnetic atoms be placed in a nonmagnetic matrix so that the number of granules $N_{g}$ is great $\left(N_{g} \gg 1\right.$ ), each of them contains $N$ magnetic atoms with Ising spins $\sigma_{i}(n)= \pm 1$, where $i$ is granular number and $n$ is the spin number inside the granule. We assume 


\section{E. E. KOKORINA, M. V. MEDVEDEV}

that all the granules contain an equal number of atoms $N$, but in general case the granules are distributed in a random way, and intragranular exchange $J_{i j}(n, m)$ is random. The Ising Hamiltonian for this system can be written as

$$
\begin{aligned}
H(\{\sigma\}) & =-\frac{1}{2} \sum_{i=1}^{N_{g}} \frac{I}{N} \sum_{n=1}^{N} \sum_{m=1, n \neq m}^{N} \sigma_{i}(n) \sigma_{i}(m) \\
& -\frac{1}{2} \sum_{i=1}^{N_{g}} \sum_{j=1, i \neq j}^{N_{g}} \sum_{n=1}^{N} \sum_{m=1}^{N} J_{i j}(n, m) \sigma_{i}(n) \sigma_{j}(m) \\
& -h_{e} \sum_{i=1}^{N_{g}} \sum_{n=1}^{N} \sigma_{i}(n) .
\end{aligned}
$$

In the first term of the Hamiltonian, which takes into account only the intragranular interactions, $I>0$ is the parameter of the intragranule ferromagnetic exchange. We accept the model of interaction among all spins and following [5] we introduce the normalizing factor $1 / N$ for intergranular exchange energy per magnetic atom of the system to be independent of granular size. The second term describes the random intergranular interaction among all spins of different granules and for simplification we assume $J_{i j}(n, m)=J_{i, j}$ that means that random interaction $J_{i j}$ depends only on the locations of centers of granules $i$ and $j$, but does not depend on the location of $n$ and $m$ sites inside granules. That model means, that the exchange parameter $J_{i j}$ weakly depends on distances comparable with the granular size. And in the third term, which responsible for the Zeeman interaction, we signify $h_{e}=g \mu_{B} H_{a}$.

Later we shall consider only the case when exchange energy of atom with its neighborhood in granule is much greater then its exchange energy with atoms of another granule. Hence, at temperature decreasing first a local ferromagnetic ordering appears inside granules and then, at a lower temperature, a long-range ferromagnetic order is established over the whole sample.

Let us take into account, that the local magnetic order can appear on every granule, but at the same time the local magnetization of the granule $i$ can be directed parallel or antiparallel to the $O Z$-axis of the laboratory coordinate system. Therefore in each granule we will use a local coordinate system, the $O Z_{i}$-axis of which rigidly connected with the direction of the local magnetization of the granule. Then instead of Ising variables $\sigma_{i}(n)$ in the laboratory coordinate system we can use new variables $\mu_{i}(n)$ in the local coordinate system with the help of the following equation:

$$
\sigma_{i}(n)=\mu_{i}(n) \cos \left(O \mathbf{Z , O} \mathbf{Z}_{i}\right) \equiv \mu_{i}(n) \tau_{i}
$$

where $\mu_{i}(n)$ takes the value \pm 1 in the projection onto the $O Z_{i}$-axis and $\cos \left(O \mathbf{Z , O} \mathbf{Z}_{i}\right) \equiv \tau_{i}$ is also an Ising variable: $\tau_{i}=1$, when the granule magnetization is directed along
$O Z$-axis, $\tau_{i}=-1$, when the granule magnetization is antiparallel to the $O Z$ laboratory axis.

Then, after the introduction of the local coordinate systems, the Hamiltonian (1) takes the form

$$
\begin{aligned}
H(\{\mu, \tau\}) & =-\frac{1}{2} \frac{I}{N} \sum_{i=1}^{N_{g}} \sum_{n=1}^{N} \sum_{m=1, n \neq m}^{N} \mu_{i}(n) \mu_{i}(m) \\
& -\frac{1}{2} \sum_{i=1}^{N_{g}} \sum_{j=1, i \neq j}^{N_{g}} J_{i j} \sum_{n=1}^{N} \sum_{m=1}^{N} \mu_{i}(n) \tau_{i} \mu_{j}(m) \tau_{j} \\
& -h_{e} \sum_{i=1}^{N_{g}} \sum_{n=1}^{N} \mu_{i}(n) \tau_{i}
\end{aligned}
$$

Following [1], we introduce in Hamiltonian (3) the molecular-field approximation. Representing the spin variables $\mu_{i}(n)$ and $\mu_{i}(n) \tau_{i}$ as the mean values $\left\langle\mu_{i}(n)\right\rangle$ and $\left\langle\mu_{i}(n) \tau_{i}\right\rangle$ and fluctuations above them and neglecting the fluctuation interaction, we obtain

$$
H_{M F}(\{\mu, \tau\})=\widetilde{E_{0}}-\sum_{i=1}^{N_{g}} \sum_{n=1}^{N}\left[h_{a i}(n)+h_{b i}(n) \tau_{i}\right] \mu_{i}(n)
$$

where we introduced two types of molecular fields molecular field, which acts on a given spin from the spins of its own granule

$$
h_{a i}(n)=\frac{I}{N} \sum_{m=1}^{N}\left\langle\mu_{i}(m)\right\rangle
$$

and the molecular field, which acts on this spin from the spins of other granules

$$
h_{b i}(n)=h_{e}+\sum_{j=1, j \neq i}^{N_{g}} J_{i j} \sum_{m=1}^{N}\left\langle\mu_{j}(m) \tau_{j}\right\rangle .
$$

Here we denote:

$$
\begin{aligned}
\widetilde{E_{0}} & =\frac{1}{2} \sum_{i=1}^{N_{g}} \sum_{n=1}^{N}\left\{h_{a i}(n)\left\langle\mu_{i}(n)\right\rangle\right. \\
& \left.+\left[h_{b i}(n)-h_{e}\right]\left\langle\mu_{i}(n) \tau_{i}\right\rangle\right\} .
\end{aligned}
$$

For brevity, the dimensionless molecular fields are denoted as

$$
a_{i}(n)=\beta h_{a i}(n), \quad b_{i}(n)=\beta h_{b i}(n) .
$$

Using the partition function $Z$ 


$$
Z=\operatorname{Sp}_{\{\sigma\}} \exp [-\beta H(\{\sigma\})]=\frac{1}{2^{N_{g}}} \operatorname{Sp}_{\{\mu, \tau\}} \exp [-\beta H(\{\mu \tau\})]
$$

it can be shown [1] that

$$
\begin{aligned}
& \operatorname{Sp}_{\{\mu, \tau\}}\left\{\prod_{i=1}^{N_{g}} \prod_{n=1}^{N} \exp \left[\left(a_{i}(n)+b_{i}(n) \tau_{i}\right) \mu_{i}(n)\right]\right\} \\
= & \operatorname{Sp}_{\{\tau\}}\left\{\prod_{i=1}^{N_{g}} \prod_{n=1}^{N}\left[\exp \left[a_{i}(n)+b_{i}(n) \tau_{i}\right]+\exp \left[-\left(a_{i}(n)+b_{i}(n) \tau_{i}\right)\right]\right]\right\} \\
= & 2^{N N_{g}} \prod_{i=1}^{N_{g}}\left\{\prod _ { n = 1 } ^ { N } \operatorname { c o s h } \left[\left(a_{i}(n)+b_{i}(n)\right]+\prod_{n=1}^{N} \cosh \left[\left(a_{i}(n)-b_{i}(n)\right]\right\}\right.\right.
\end{aligned}
$$

Hence, the partition function $Z$ is

$$
\begin{aligned}
& Z=e^{-\beta \widetilde{E_{0}}} 2^{N_{g}(N-1)} \prod_{i=1}^{N_{g}}\left\{K_{i}^{(+)}+K_{i}^{(-)}\right\} \\
& K_{i}^{( \pm)}=\prod_{p=1}^{N} \cosh \left[a_{i}(p) \pm b_{i}(p)\right] .
\end{aligned}
$$

Knowing $Z$, we derive the self-consistent equations for the order parameters in the local coordinate system

$$
\left\langle\mu_{i}(n)\right\rangle=\frac{\tanh \left[a_{i}(n)+b_{i}(n)\right] K_{i}^{(+)}+\tanh \left[a_{i}(n)-b_{i}(n)\right] K_{i}^{(-)}}{K_{i}^{(+)}+K_{i}^{(-)}}
$$

and in the laboratory coordinate system

$$
\left\langle\mu_{i}(n) \tau_{i}\right\rangle=\left\langle\sigma_{i}(n)\right\rangle=\frac{\tanh \left[a_{i}(n)+b_{i}(n)\right] K_{i}^{(+)}-\tanh \left[a_{i}(n)-b_{i}(n)\right] K_{i}^{(-)}}{K_{i}^{(+)}+K_{i}^{(-)}}
$$

Further simplification of (11)-(14) can be made with taking into account, that molecular field $h_{b i}(n)$ will be equal for all sites $n$ of single granule $i$ (but various for the different granules) as we consider for intergranular exchange $J_{i j}$ the dependence only from the distance between centers of granules. Moreover, all spins inside the granule are connected with each other by equal exchange interactions, and in the absence of random intergranular exchange $\left\langle\mu_{i}(n)\right\rangle$ would be equal for all sites and all granules. As a result it leads to the dependence of $\left\langle\mu_{i}(n)\right\rangle$ and $\left\langle\mu_{i}(n) \tau_{i}\right\rangle$ only on the number $i$ of granule, but no dependence on the site number $n$. We obtain

$$
a_{i}(n)=a_{i}=\beta I\left\langle\mu_{i}\right\rangle,
$$

$$
\begin{gathered}
b_{i}(n)=b_{i}=\beta\left(h_{e}+N \sum_{j=1}^{N_{g}} J_{i j}\left\langle\mu_{j} \tau_{j}\right\rangle\right) \\
K_{i}^{( \pm)}=\cosh ^{N}\left(a_{i} \pm b_{i}\right)
\end{gathered}
$$

$$
\left\langle\mu_{i}\right\rangle=\frac{\tanh \left(a_{i}+b_{i}\right) K_{i}^{(+)}+\tanh \left(a_{i}-b_{i}\right) K_{i}^{(-)}}{K_{i}^{(+)}+K_{i}^{(-)}},
$$




$$
\begin{aligned}
\left\langle\mu_{i} \tau_{i}\right\rangle & =\left\langle\sigma_{i}\right\rangle \\
& =\frac{\tanh \left(a_{i}+b_{i}\right) K_{i}^{(+)}-\tanh \left(a_{i}-b_{i}\right) K_{i}^{(-)}}{K_{i}^{(+)}+K_{i}^{(-)}}
\end{aligned}
$$

\section{BOUNDARIES OF THE STABILITY FOR THE SUPERPARAMAGNETIC STATE}

At first we consider particular case $J_{i j}=0$. In the absence of random intergranular exchange the molecular fields $b_{i}(8)$ are equal for all granules for the reason of depending only on the uniform magnetic field $h_{e}=g \mu_{B} H_{a}$. Then $\left\langle\mu_{i}\right\rangle$ and $\left\langle\sigma_{i}\right\rangle$ are also equal for all granules, and, omitting $i$, in the case of weak external field $b=\beta h_{e} \ll 1$ from (11) and (18) we can get:

$$
\begin{gathered}
\langle\mu\rangle \approx \tanh a+O\left(b^{2}\right) \\
\langle\sigma\rangle=\langle\mu \tau\rangle \approx\left[1+(N-1) \tanh ^{2} a\right] b+O\left(b^{3}\right) .
\end{gathered}
$$

From (19) follows, that at $b=0$ spontaneous thermodynamic mean value $\langle\mu\rangle_{H_{a}=0} \equiv \mu_{0}$ is given by well known Ising equation

$$
\mu_{0}=\tanh a_{0}=\tanh \left(\beta I \mu_{0}\right)
$$

with the critical temperature of the appearance of the local magnetic order $T_{c 1}$

$$
k_{B} T_{c 1}=I
$$

and temperature dependencies of local magnetic order parameter

$$
\begin{gathered}
\mu_{0} \cong\left[3\left(1-\frac{T}{T_{c 1}}\right)\right]^{1 / 2} \text { at } 1-\frac{T}{T_{c 1}} \ll 1, \\
\mu_{0} \cong 1-2 \exp \left(-\frac{2 I}{k_{B} T}\right)=1-2 \exp \left(-\frac{2 T_{c 1}}{T}\right) \\
\text { at } \frac{T}{T_{c 1}} \ll 1 .
\end{gathered}
$$

An initial paramagnetic susceptibility per granule can be derived from (20)

$$
\chi_{0}=\left.N g \mu_{B} \frac{d}{d H_{a}}\langle\sigma\rangle\right|_{H_{a}=0}=\frac{N g^{2} \mu_{B}^{2}\left[1+(N-1) \mu_{0}^{2}\right]}{k_{B} T}
$$

which in the limit $I \rightarrow \infty$ or $\mu_{0}=1$ leads to the case of rigid superparamagnetic moment with $\chi_{0}=$ $N^{2} g^{2} \mu_{B}^{2} / k_{B} T$.

Before investigating the general case of taking into account intergranular and intragranular interactions simultaneously, we consider an instructive particular case, in which intragranular interaction is completely neglected $I=0$. Then from (15) follows $a_{i}=0$ and $\left\langle\mu_{i}\right\rangle=0$, it means that no local ferromagnetic order exists at $I=0$ on the granule with the fluctuating axis direction for this ordering. Now the granules consist of $N$ noninteracting spins that are connected with each other only by intergranular exchange $J_{i j}$, and equations for $\left\langle\sigma_{i}\right\rangle$ (18) take the form

$$
\langle\sigma\rangle=\tanh b_{i}=\tanh \left[\beta\left(h_{e}+N \sum_{j} J_{i j}\left\langle\sigma_{j}\right\rangle\right)\right]
$$

We choose a Gaussian random distribution model for intergranular interaction

$$
P\left(J_{i j}\right)=\frac{1}{\left[2 \pi D\left(J_{i j}\right)\right]^{1 / 2}} \exp \left[-\frac{\left(J_{i j}-\bar{J}_{i j}\right)^{2}}{2 D\left(J_{i j}\right)}\right],
$$

where a mean value of random exchange integrate $\bar{J}_{i j}$ (the overline means an averaging over disorder) and its dispersion $D\left(J_{i j}\right)$ are normalized in the following way:

$$
\bar{J}_{i j}=\frac{1}{N N_{g}} J_{0}, \quad D\left(J_{i j}\right)=\frac{1}{N^{2} N_{g}} J^{2}
$$

Then we can get

$$
\overline{b_{i}}=\beta\left(h_{e}+J_{0} \sigma\right)
$$

$$
\overline{b_{i}^{2}}=\beta^{2}\left[\left(h_{e}+J_{0} \sigma\right)^{2}+J^{2} q\right]
$$

where

$$
\sigma=\overline{\left\langle\sigma_{j}\right\rangle}, \quad q=\overline{\left\langle\sigma_{j}\right\rangle^{2}}
$$

( $\sigma$ is ferromagnetic order parameter over the whole sample and $q$ is Edwards-Anderson parameter of spin-glass order [4]). It is seen that with such normalization of distribution parameters (27) the mean values of dimensionless molecular field $\overline{b_{i}}$ and its squared value $\overline{b_{i}^{2}}$ do not depend on the system size and are coincident with corresponding expressions for these values in the SherringtonKirkpatrick model [4] (at $N=1$ - one spin per granule - (27) and (28) turn to the formulas of [4]).

Therefore in the case $I=0$, as in [4], at $J_{0}>J$ the system from ordinary paramagnetic state would turn to the global ferromagnetic order over the whole sample $\sigma \neq 0$, $q \neq 0$ at temperature 


$$
k_{B} T_{c 0}=J_{0}
$$

and at $J_{0}<J$ - to the spin glass order with $\sigma=0, q \neq 0$ at temperature

$$
k_{B} T_{s g 0}=J
$$

(with one peculiarity: according to our model of intergranular interactions, all spins inside an individual granule would be effected by the same random intergranular field, that gives the same random direction for all spins inside granule in the spin-glass state).

Let us now consider the case, when the global magnetic order over the whole sample appears on the base of established local ferromagnetic order $\mu_{0} \neq 0$ on granules, and we shall assume, that $I \gg J_{0}, J$. It means that the temperature of appearance of a local ferromagnetic order $T_{c 1}(22)$ is much higher than "bare" temperatures of the global magnetic order $T_{c 0}(32)$ or $T_{s g 0}(33)$.

Making expansions of (17) and (18) in powers of $b_{i} \ll 1$, we get

$$
\begin{gathered}
\left\langle\mu_{i}\right\rangle \cong \tanh a_{i}+\Gamma_{1}(i) b_{i}^{2}-\Gamma_{2}(i) b_{i}^{4}+\ldots, \\
\left\langle\sigma_{i}\right\rangle \equiv\left\langle\mu_{i} \tau_{i}\right\rangle \cong A_{1}(i) b_{i}-A_{2}(i) b_{i}^{3}+A_{3}(i) b_{i}^{5}-\ldots,
\end{gathered}
$$

where

$$
\begin{aligned}
\Gamma_{1}(i) & =(N-1) \tanh a_{i}\left(1-\tanh a_{i}^{2}\right), \\
\Gamma_{2}(i) & =\frac{1}{3}(N-1) \tanh a_{i}\left(1-\tanh ^{2} a_{i}\right) \\
& \times\left[2+\left(N^{2}+N-3\right) \tanh ^{2} a_{i}\right]
\end{aligned}
$$

and

$$
\begin{aligned}
& A_{1}(i)=1+(N-1) \tanh ^{2} a_{i}, \\
& A_{2}(i)=\frac{1}{3}\left[1+4(N-1) \tanh ^{2} a_{i}\right. \\
& \left.+(N-1)\left(N^{2}+N-3\right) \tanh ^{4} a_{i}\right] \\
& A_{3}(i)=\frac{1}{15}\left[2+17(N-1) \tanh ^{2} a_{i}\right. \\
& +10(N-1)\left(N^{2}+N-3\right) \tanh ^{4} a_{i} \\
& \left.+(N-1)\left(2 N^{4}+2 N^{3}-8 N^{2}-8 N+15\right) \tanh ^{6} a_{i}\right] .
\end{aligned}
$$

At $I=0, \quad \tanh a_{i}=0$ coefficients $(37)$ turn to the coefficients of $\tanh b_{i}$ expansion in powers of $b_{i}: A_{1}=1, A_{2}=$ $\frac{1}{3}$ and $A_{3}=\frac{2}{15}$, and in the limit $I \rightarrow \infty, \quad \tanh a_{i} \rightarrow 1$ turn to $A_{1}=N, A_{2}=\frac{1}{3} N^{3}$ and $A_{3}=\frac{2}{15} N^{5}$.

For further solution of expansions (34) and (35) should be averaged over disorder. For a more complex analysis of this problem the replica method should be used [4] for calculating the free energy of a system on the basis of Hamiltonian (3). But we restrict ourselves by a more simple approach, based on the usage of averaging process with Gaussian distribution of random molecular fields. This approach (as shown in [6]) in the temperature region over the de Almeida-Thouless instability line [7] in spin glass leads to the same results as the replicasymmetric method [4].

Taking into account, that $b_{i}(15)$ consists of $N \gg 1$ random parts, we can get a distribution function with the help of saddle-point method (see also [6])

$$
P\left(b_{i}\right)=\frac{1}{\left(2 \pi \beta^{2} J^{2} q\right)^{1 / 2}} \exp \left\{-\frac{\left[b_{i}-\beta\left(h_{e}+J_{0} \sigma\right)\right]^{2}}{2 \beta^{2} J^{2} q}\right\} .
$$

As for the random intragranular molecular field $a_{i}=$ $\beta I\left\langle\mu_{i}\right\rangle(15)$, at $h_{e}=0$ and in the temperature interval above the appearance of the spontaneous global magnetic order the values $\left\langle\mu_{i}\right\rangle$ and $a_{i}$ are equal for all granules that means they are not random. The "switching on" of the magnetic field $h_{e} \neq 0$ or lowering of temperature leads to the occurrence of random part in $\left\langle\mu_{i}\right\rangle$. But these additional terms to $\left\langle\mu_{i}\right\rangle$ in (17) cannot be expressed by a simple sum of the $N_{g} \gg 1$ terms from the granules $j \neq i$, which could give us an opportunity to get the distribution function $P\left(a_{i}\right)$. But we may take into account, that because of conditions $I \gg J_{0}, J$ a relative contribution from the intergranular interactions in the formation of $\left\langle\mu_{i}\right\rangle$ can be much less then the contribution from the regular intragranular interactions. That is why we can suppose, that deviation of $\mu_{i}$ from the mean value $\mu \equiv \bar{\mu}_{i}$ will not be large. That is the reason for taking the simplest approximation, that at averaging over disorder we can replace everywhere random $\mu_{i}$ and $a_{i}$ with their mean values $a \equiv \bar{a}_{i}$ and $\mu \equiv \bar{\mu}_{i}$.

Finally, after averaging expansions of $\left\langle\mu_{i}\right\rangle$ (34) and $\left\langle\sigma_{i}\right\rangle(35)$, and also of $\left\langle\sigma_{i}\right\rangle^{2}$ over disorder, we obtain

$$
\begin{aligned}
& \mu=\tanh a+\Gamma_{1} \beta^{2}\left[\left(h_{e}+J_{0} \sigma\right)^{2}+J^{2} q\right] \\
& -\Gamma_{2} \beta^{4}\left[\left(h_{e}+J_{0} \sigma\right)^{4}+6\left(h_{e}+J_{0} \sigma\right)^{2} J^{2} q+3 J^{4} q^{2}\right]+\ldots
\end{aligned}
$$

$$
\begin{aligned}
\sigma & =A_{1} \beta\left(h_{e}+J_{0} \sigma\right)-A_{2} \beta^{3}\left[\left(h_{e}+J_{0} \sigma\right)^{3}\right. \\
& \left.+3\left(h_{e}+J_{0} \sigma\right) J^{2} q\right]+A_{3} \beta^{5}\left[\left(h_{e}+J_{0} \sigma\right)^{5}\right. \\
& \left.+10\left(h_{e}+J_{0} \sigma\right)^{3} J^{2} q+15\left(h_{e}+J_{0} \sigma\right) J^{4} q^{2}\right]-\ldots
\end{aligned}
$$

$$
\begin{aligned}
& q=A_{1}^{2} \beta^{2}\left[\left(h_{e}+J_{0} \sigma\right)^{2}+J^{2} q\right] \\
& -2 A_{1} A_{2} \beta^{4}\left[\left(h_{e}+J_{0} \sigma\right)^{4}+6\left(h_{e}+J_{0} \sigma\right)^{2} J^{2} q+3 J^{4} q^{2}\right]
\end{aligned}
$$




$$
\begin{aligned}
& +\left(A_{2}^{2}+2 A_{1} A_{3}\right) \beta^{6}\left[\left(h_{e}+J_{0} \sigma\right)^{6}+15\left(h_{e}+J_{0} \sigma\right)^{4} J^{2} q\right. \\
& \left.+45\left(h_{e}+J_{0} \sigma\right)^{2} J^{4} q^{2}+15 J^{6} q^{3}\right]-\ldots
\end{aligned}
$$

\section{A. The temperature of spontaneous transition to the state with the global magnetic order}

In the absence of applied field $\left(h_{e}=0\right)$ from (40) and (41) we get linearized equations for the order parameters $\sigma_{0}$ and $q_{0}$ :

$$
\left\{\begin{array}{l}
\sigma_{0}=A_{1} \beta J_{0} \sigma_{0} \\
q_{0}=A_{1}^{2} \beta^{2}\left[J_{0}^{2} \sigma_{0}^{2}+J^{2} q_{0}\right]
\end{array}\right.
$$

(index 0 marks order parameters $\sigma_{0}$ and $q_{0}$, obtained at $\left.h_{e}=0\right)$.

Hence, the temperature of thr transition to the state with spontaneous magnetization throughout the sample $\sigma_{0} \neq 0, q_{0} \neq 0$ (let us call it superferromagnetic, following [8]) can be derived from the equation

$$
1=\left[1+(N-1) \tanh ^{2} a_{0}\left(\beta_{c 2}\right)\right] \beta_{c 2} J_{0}
$$

but the transition temperature to the state without spontaneous magnetization $\sigma_{0}=0, q_{0} \neq 0$ (let us call it macrospin glass) can be derived from the equation

$$
1=\left[1+(N-1) \tanh ^{2} a_{0}\left(\beta_{m s g}\right)\right] \beta_{m s g} J
$$

At the moment when the global ordering appears, intergranular molecular fields are infinitely small, and then $a_{0}\left(\beta_{c r}\right)$, where $\beta_{c r}=\beta_{c 2}$ or $\beta_{m s g}$, is defined only by regular intragranular interactions:

$$
\mu_{0}\left(\beta_{c r}\right)=\tanh a_{0}\left(\beta_{c r}\right)=\tanh \left[\beta_{c r} I \mu_{0}\left(\beta_{c r}\right)\right]
$$

Then (43) and (44) can be rewritten as

$$
\begin{aligned}
& 1=\left[1+(N-1) \tilde{\mu}^{2}\right] \beta_{c 2} J_{0}, \\
& 1=\left[1+(N-1) \tilde{\mu}^{2}\right] \beta_{m s g} J
\end{aligned}
$$

(here we signify $\tilde{\mu} \equiv \mu_{0}\left(\beta_{c r}\right)$ ).

Equations (46) and (47) can be easily solved, with the usage for $\tilde{\mu}$ of a well-known relation (23) in the case $1-\left(T_{c r} / T_{c 1}\right) \ll 1$ or $(24)$ at $T_{c r} / T_{c 1} \ll 1$. Assuming that $T_{c 2}$ lies in the temperature range, where $\mu_{0}(T)$ is described by the relation (23), we obtain

$$
T_{c 2}=\frac{1}{1+\left(k_{B} T_{c 1}-J_{0}\right) /(3 N-2) J_{0}} T_{c 1}
$$

$$
\approx \frac{1}{1+k_{B} T_{c 1} / 3 N J_{0}} T_{c 1}
$$

If the following condition $3 N J_{0} \gg I=k_{B} T_{c} \gg J_{0}$ is true, then

$$
T_{c 2} \approx\left(1-I / 3 N J_{0}\right) T_{c 1}
$$

The validity of equation $3 N J_{0} \gg I \gg J_{0}$ means also the validity $N \tilde{\mu}^{2} \gg 1$, and in this case equations (46) and (47) turns to

$$
1 \cong N \tilde{\mu}^{2} \beta_{c 2} J_{0}, \quad 1 \cong N \tilde{\mu}^{2} \beta_{m s g} J
$$

In the same way we obtain

$$
T_{m s g} \approx \frac{1}{1+k_{B} T_{c 1} / 3 N J} T_{c 1} \approx\left(1-\frac{I}{3 N J}\right) T_{c 1}
$$

at $3 N J \gg I \gg J$

If $T_{c 2}$ or $T_{m s g}$ falls in the temperature range, where $\tilde{\mu}$ have the exponential form (24), then

$$
k_{B} T_{c 2} \approx N J_{0}\left(1-4 e^{-2 I / N J_{0}}\right),
$$

$$
k_{B} T_{m s g} \approx N J\left(1-4 e^{-2 I / N J}\right),
$$

that will take place at $I \gg N J_{0} \gg J_{0}$ and $I \gg N J \gg J$.

Comparing (46) and (47) we see that at $J_{0}>J$ with decreasing temperature the system exhibits the transition from the superparamagnetic state to the superferromagnetic one, and at $J_{0}<J$ the system passes into the macrospin glass state.

\section{B. Linear and nonlinear magnetic susceptibility of superparamagnetic state}

A magnetization of the granule $M_{g}$ in the applied field $H_{a}$ at temperatures above the critical ones $T_{c 2}$ and $T_{m s q}$ can be expressed in the form

$$
M_{g}\left(H_{a}\right)=N g \mu_{B} \sigma\left(H_{a}\right)=\chi_{0} H_{a}+\chi_{n l} H_{a}^{3}+\ldots
$$

In order to find linear $\chi_{0}$ and nonlinear $\chi_{n l}$ magnetic susceptibilities, let us solve equations (39)-(41), putting the expansions of $\mu, \sigma$ and $q$ in the form:

$$
\begin{gathered}
\mu=\mu_{0}+\mu_{1} h_{e}^{2}+\ldots, \quad \sigma=\sigma_{1} h_{e}+\sigma_{3} h_{e}^{3}+\ldots \\
q=q_{1} h_{e}^{2}+\ldots
\end{gathered}
$$

For the averaged linear susceptibility $\chi_{0}$ (per one granule) we find 


$$
\chi_{0}=N g^{2} \mu_{B}^{2} \sigma_{1}=N g^{2} \mu_{B}^{2} \beta \frac{1+(N-1) \mu_{0}^{2}}{1-\left[1+(N-1) \mu_{0}^{2}\right] \beta J_{0}} .
$$

Thus, at $T>T_{c 1}$ and $\mu_{0}=0$ an expression (56) turns to be a Curie-Weiss susceptibility for $N$ spins:

$$
\chi_{0}=N g^{2} \mu_{B}^{2}\left(k_{B} T-J_{0}\right)^{-1} \quad \text { at } \quad T>T_{c 1}
$$

with pole, having been projecting on the "bare" Curie temperature $k_{B} T_{c 0}=J_{0}$. In the case of transition over $T_{c 1}$ and the occurrence of the local ferromagnetic order in the granule the pole of $\chi_{0}$ is shifted to the point $T_{c 2}$, which is defined by equation (46).

Assuming that the critical temperature of transition to superferromagnetic state $T_{c 2}$ is rather low in com- parison with $T_{c 1}$, so that on the large part of temperature range of superparamagnetism existence the condition $N \mu_{0}^{2}(T) \gg 1$ is valid, then $\chi_{0}$ in this region can be expressed in the form

$$
\chi_{0} \simeq \frac{\left[N g \mu_{B} \mu_{0}(T)\right]^{2}}{k_{B} T-N \mu_{0}^{2}(T) J_{0}} \quad \text { at } \quad N \mu_{0}^{2}(T) \gg 1
$$

and approaching $T_{c 2}$ from above

$$
\chi_{0} \simeq \frac{\left[N g \mu_{B} \tilde{\mu}\right]^{2}}{\left(1-2\left(\widetilde{\mu}^{\prime} / \tilde{\mu}\right) T_{c 2}\right) k_{B}\left(T-T_{c 2}\right)}
$$

Here $\tilde{\mu}^{\prime}=\left.\frac{d}{d T} \mu_{0}(T)\right|_{T=T_{c 2}}$.

For the cases $1-T_{c 2} / T_{c 1} \ll 1$ and $T_{c 2} / T_{c 1}$ we get

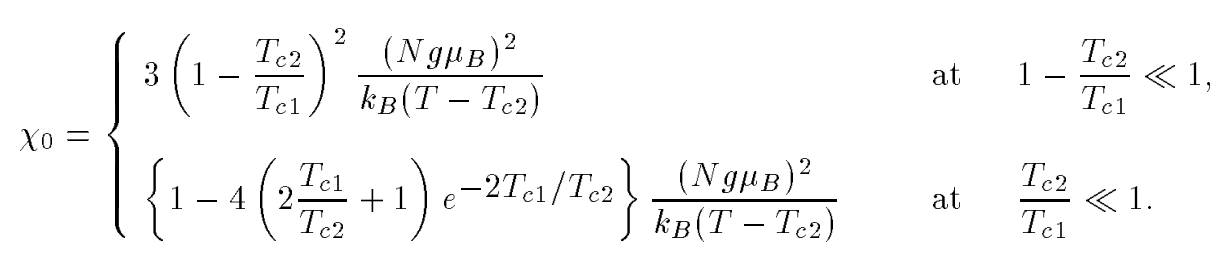

If $J>J_{0}$ and $T_{m s g}>T_{c 2}$, then as the temperature decreases $\chi_{0}$ can not get the pole, and at $T=T_{m s g}$ a cusp occurs in the temperature behaviour of $\chi_{0}(T)$, so that at the point $T=T_{m s g}$ we obtain

$$
\chi_{0}\left(T_{m s g}\right)=N g^{2} \mu_{B}^{2} \frac{1}{J-J_{0}} .
$$

As for the nonlinear susceptibility $\chi_{n l}$, we find in the superparamagnetic region that

$$
\chi_{n l}=N g^{4} \mu_{B}^{4} \sigma_{3} \approx \frac{N^{3} g^{4} \mu_{B}^{4}}{\left(1-N \mu_{0}^{2} \beta J_{0}\right)^{4}\left(1-N^{2} \mu_{0}^{4} \beta^{2} J^{2}\right)}\left\{-\frac{1}{3} N \mu_{0}^{4} \beta^{3}\left(1+2 N^{2} \mu_{0}^{4} \beta^{2} J^{2}\right)+\frac{2 \mu_{0}^{2}\left(1-\mu_{0}^{2}\right)^{2} \beta^{4} I}{1-\left(1-\mu_{0}^{2}\right) \beta I}\right\}
$$

(as a general expression is too unwieldy we represent a result which is valid only in the temperature range where $\left.N \mu_{0}^{2}(T) \gg 1\right)$.

It can be easily seen that $\chi_{n l}$ will be singular at the transition as to superferromagnetic state as well to the macrospin glass state.

If $J_{0}>J$ and we have a transition at the temperature $T_{c 2}$, expressed by condition $1 \simeq N \widetilde{\mu}^{2} \beta_{c 2} J_{0}$, then in the neighborhood of the point $T_{c 2}$ we find

$$
\chi_{n l} \cong \frac{N^{3} g^{4} \mu_{B}^{4}}{1-\left(J / J_{0}\right)^{2}} \frac{1}{\left(1-2\left(\widetilde{\mu}^{\prime} / \widetilde{\mu}\right) T_{c 2}\right)^{4}} \frac{k_{B} T_{c 2}}{\left[k_{B}\left(T-T_{c 2}\right)\right]^{4}}\left\{-\frac{1}{3} N \widetilde{\mu}^{4}\left[1+2\left(J / J_{0}\right)^{2}\right]+\frac{2 \widetilde{\mu}^{2}\left(1-\tilde{\mu}^{2}\right)^{2} \beta_{c 2} I}{1-\left(1-\widetilde{\mu}^{2}\right) \beta_{c 2} I}\right\} .
$$

Thus in the case $1-T_{c 2} / T_{c 1} \ll 1$ it can be expressed as

$$
\chi_{n l} \approx-3\left(N g \mu_{B}\right)^{4} \frac{J_{0}^{2}+2 J^{2}}{J_{0}^{2}-J^{2}}\left(1-T_{c 2} / T_{c 1}\right)^{6} \frac{k_{B} T_{c 2}}{\left[k_{B}\left(T-T_{c 2}\right)\right]^{4}}
$$

and in the case $T_{c 2} / T_{c 1} \ll 1$ as 


$$
\chi_{n l} \approx-\frac{1}{3}\left(N g \mu_{B}\right)^{4} \frac{J_{0}^{2}+2 J^{2}}{J_{0}^{2}-J^{2}}\left[1-8\left(4 T_{c 1} / T_{c 2}+1\right) e^{-2 T_{c 1} / T_{c 2}}\right] \frac{k_{B} T_{c 2}}{\left[k_{B}\left(T-T_{c 2}\right)\right]^{4}}
$$

At $J>J_{0}$ in $(62)$ the pole appears at first at $1-N \tilde{\mu}^{2} \beta_{m s g} J=0(50)$. Then in the vicinity of $T_{m s g}$ it will be

$$
\chi_{n l} \cong \frac{N^{3} g^{4} \mu_{B}^{4}}{2\left(1-\left(J / J_{0}\right)\right)^{4}} \frac{1}{\left(1-2\left(\widetilde{\mu}^{\prime} / \widetilde{\mu}\right) T_{m s g}\right)} \frac{1}{k_{B}^{3} T_{m s g}^{2}\left(T-T_{m s g}\right)}\left\{-N \widetilde{\mu}^{4}+\frac{2 \widetilde{\mu}^{2}\left(1-\widetilde{\mu}^{2}\right)^{2} \beta_{m s g} I}{1-\left(1-\widetilde{\mu}^{2}\right) \beta_{m s g} I}\right\}
$$

so at $1-T_{m s g} / T_{c 1} \ll 1$ we get

$$
\chi_{n l}=-\frac{9}{2}\left(N g \mu_{B}\right)^{4}\left(\frac{J}{J-J_{0}}\right)^{4}\left(1-T_{m s g} / T_{c 1}\right)^{3} \frac{1}{k_{B}^{3} T_{m s g}^{2}\left(T-T_{m s g}\right)}
$$

and at $T_{m s g} / T_{c 1} \ll 1$ we find

$$
\chi_{n l}=-\frac{1}{2}\left(N g \mu_{B}\right)^{4}\left(\frac{J}{J-J_{0}}\right)^{4}\left[1-8\left(T_{c 1} / T_{m s g}+1\right) e^{-2 T_{c 1} / T_{m s g}}\right] \frac{1}{k_{B}^{3} T_{m s g}^{2}\left(T-T_{m s g}\right)} .
$$

It can be shown that, as well as at transition to the spin glass state $[9,10]$, nonlinear susceptibility at the approach to the macrospin glass state from above tends to $-\infty$.

\section{THE DE ALMEIDA-THOULESS INSTABILITY LINE}

The de Almeida-Thouless instability line [7] means the appearance of the nonergodic region on the phase diagram of a disordered magnet (see, for example, [6]). In the limit of nonzero magnetic field $H_{a}$ in the Ising model for Sherrington-Kirkpatrick spin glass this line coincide with the temperature transition line $T_{s g}$ into the spin glass phase, and at $H_{a} \neq 0$, when the system starts magnetized, the de Almeida-Thouless line $T_{A T}$ shifted lower then temperatures $T_{s g}[6]$.

One of the easiest way to reveal the de AlmeidaThouless line is the following. If we calculate the mean square value of one-site magnetic susceptibility $\overline{\chi_{i}^{2}}$ with the help of such methods, which do not take into account the complicated intrinsic structure of spin glass phase (like the symmetrical replica method [4] or like heuristic methods [6]), then in a certain field and temperature region the condition $\overline{\chi_{i}^{2}}>0$ will be broken. Let us use this method in our problem.

We shall consider a random one-site magnetic susceptibility in the following way:

$$
\chi_{i}=g \mu_{B} \frac{d\left\langle\sigma_{i}\right\rangle}{d H_{a}}
$$

Then, taking derivations for all terms of expansion $\left\langle\sigma_{i}\right\rangle$
(35), we get

$$
\begin{aligned}
\chi_{i} & =g^{2} \mu_{B}^{2}\left\{\left[\frac{d}{d H_{a}} A_{1}(i)\right] b_{i}-\left[\frac{d}{d H_{a}} A_{2}(i)\right] b_{i}^{3}\right. \\
& \left.+\left[\frac{d}{d H_{a}} A_{3}(i)\right] b_{i}^{5}-\ldots\right\} \\
& +\left[A_{1}(i)-3 A_{2}(i) b_{i}^{2}+5 A_{3}(i) b_{i}^{4}-\ldots\right] \\
& \times \beta\left(g^{2} \mu_{b}^{2}+N \sum_{j=1}^{N_{g}} J_{i j} \chi_{j}\right) .
\end{aligned}
$$

Squaring both the sides of (70) and making averaging over disorder, we find

$$
\begin{aligned}
\overline{\chi_{i}^{2}} & =\Phi\left\{1-\beta^{2} J^{2}\left[A_{1}^{2}-6 A_{1} A_{2} \overline{b_{i}^{2}}\right.\right. \\
& \left.\left.+\left(9 A_{2}^{2}+10 A_{1} A_{2}\right) \overline{b_{i}^{4}}-\ldots\right]\right\}^{-1}
\end{aligned}
$$

(where we do not give a general form of numerator $\Phi$ because of its insignificance), and the denominator because of $\overline{\chi_{i}^{2}}>0$ should satisfy the condition

$$
\begin{aligned}
& 1-\beta^{2} J^{2}\left[A_{1}^{2}-6 A_{1} A_{2} \overline{b_{i}^{2}}\right. \\
& \left.+\left(9 A_{2}^{2}+10 A_{1} A_{3}\right) \overline{b_{i}^{4}}-\ldots\right]>0 .
\end{aligned}
$$


Inequality (72) is a high-temperature expansion of the de Almeida-Thouless stable condition which is generalized for case of disordered granular Ising magnet. At $I=0$ (or $\tanh a=0$ ) it turns to usual de Almeida-Thouless condition [7]:

$$
1-\beta^{2} J^{2} \overline{\operatorname{sech}^{4} b_{i}}
$$

$$
\approx 1-\beta^{2} J^{2}\left(1-2 \overline{b_{i}^{2}}+7 / 3 \overline{b_{i}^{4}}-\ldots\right)>0
$$

Let us calculate the de Almeida-Thouless line for the case $J>J_{0}$ assuming that it lies in the temperature range, where the following assumption $N \tilde{\mu}^{2} \sim$ $N \tanh ^{2} a \gg 1$ is valid. Then, leaving in the coefficients $A_{1}, A_{2}, A_{3}(37)$ the largest contributions and taking in $\overline{b_{i}^{2}}$ and $\overline{b_{i}^{4}}$ contributions $\sim h_{e}^{2}$, we get

$1-\beta^{2} J^{2} N^{2} \tanh ^{4} a\left\{1-2 \beta^{2} N^{2} \tanh ^{2} a\left[\left(J_{0} \sigma+h_{e}\right)^{2}+J^{2} q\right]+7 \beta^{4} N^{4} \tanh ^{4} a\left[2\left(J_{0} \sigma+h_{e}\right)^{2} J^{2} q+J^{4} q^{2}\right]-\ldots\right\}>0$

At temperatures $T \leq T_{m s g}$ with the help of equations (39)-(41) we obtain $q, \sigma$ and also $\mu$, correct to amount $\sim h_{e}^{2}$ :

$$
\begin{aligned}
& q=\tilde{\mu}^{2} \frac{1+p\left(1-\tilde{\mu}^{2}\right)}{1-p\left(1-\widetilde{\mu}^{2}\right)} t+\frac{1}{3} \widetilde{\mu}^{2} \frac{1+p(7-6 p)\left(1-\widetilde{\mu}^{2}\right)+2 p^{2}\left(1-\widetilde{\mu}^{2}\right)^{2}-4 p^{3}\left(1-\widetilde{\mu}^{2}\right)^{3}}{\left(1-p\left(1-\widetilde{\mu}^{2}\right)\right)^{3}} t^{2}+\ldots \\
& +\frac{1}{\left(J-J_{0}\right)^{2}} \frac{1-p\left(1-\tilde{\mu}^{2}\right)}{1+p\left(1-\widetilde{\mu}^{2}\right)} \frac{h_{e}^{2}}{2 t}+\ldots, \\
& \sigma \simeq \frac{1}{J-J_{0}} h_{e}+O\left(h_{e}^{3}\right) \\
& \mu=\tilde{\mu}+\tilde{\mu}\left(1-\tilde{\mu}^{2}\right) \frac{p}{1-p\left(1-\tilde{\mu}^{2}\right)} t+\tilde{\mu}\left(1-\tilde{\mu}^{2}\right) \frac{p(1-p)}{\left(1-p\left(1-\tilde{\mu}^{2}\right)\right)^{3}} t^{2}+\ldots \\
& +\frac{\left(1-\widetilde{\mu}^{2}\right)}{N \widetilde{\mu}^{3}} \frac{1}{\left(J-J_{0}\right)^{2}} \frac{1}{1+p\left(1-\tilde{\mu}^{2}\right)} \frac{h_{e}^{2}}{2 t}+\ldots,
\end{aligned}
$$

where we denote $t=1-T / T_{m s g}$ and $p \equiv \beta_{m s g} I$.

Putting (75)-(77) in the left part of (74), neglecting terms $\sim h_{e}^{2}$ of $\mu$ in comparison with analogous terms of $q$ (it means a more severe requirement $N \widetilde{\mu}^{3} \gg 1$ ) and equating this result to zero, we get an equation for the de Almeida-Thouless temperature $T_{A T}$ :

$$
\frac{4}{3} \tilde{\mu}^{2}\left[\frac{1+\left(1-\tilde{\mu}^{2}\right) \beta_{m s g} I}{1-\left(1-\tilde{\mu}^{2}\right) \beta_{m s g} I}\right]^{3} t_{A T}^{3}=\left(\frac{g \mu_{B} H_{a}}{J-J_{0}}\right)^{2}
$$

(here $t_{A T}=1-T_{A T} / T_{m s g}$ ).

If $1-T_{m s g} / T_{c 1} \ll 1$ is valid, then using for $\tilde{\mu} \equiv$ $\mu_{0}\left(T_{m s g}\right)$ an expression (23), we find

$$
t_{A T}^{3}=\frac{1}{4}\left(1-\frac{T_{m s g}}{T_{c 1}}\right)^{2}\left(\frac{g \mu_{B} H_{a}}{J-J_{0}}\right)^{2}
$$

On another condition, when $T_{c 1} \gg T_{m s g}$ and $\tilde{\mu} \approx 1$, it follows

$$
t_{A T}^{3}=\frac{3}{4}\left[1-4\left(6 \frac{T_{c 1}}{T_{m s g}}-1\right) e^{-2 T_{c 1} / T_{m s g}}\right]\left(\frac{g \mu_{B} H_{a}}{J-J_{0}}\right)^{2} .
$$

Let us compare these results with the familiar result of de Almeida-Thouless [7]

$$
t_{A T}^{3}=\frac{3}{4}\left(\frac{g \mu_{B} H_{a}}{J-J_{0}}\right)^{2}
$$

which can be obtained in our model in the case of ignoring the intragranular interactions $(I=0)$. It can be seen, that at concrete $H_{a}, J$ and $J_{0}$ the lower is the average value of $\tilde{\mu}$, composing the spontaneous local granular magnetization in the moment of spin-glass appearing, the wider is a relative width of stability region $t_{A T}$. And only in the limit $I \rightarrow \infty$ or $T_{c 1} \rightarrow \infty$, as it can be seen from $(80)$, a relative width of temperature stable region $t_{A T}$ will coincide with (94). Of course, we should take into 


\section{E. E. KOKORINA, M. V. MEDVEDEV}

account that in this limit $I \rightarrow \infty$ the transition temperature itself $k_{B} T_{m s g}(I \rightarrow \infty)=N J$ (see $\left.(53)\right)$ is $N$ times greater, then the "bare" temperature $k_{B} T_{s g 0}=J(33)$, obtained at $I=0$.

Let us note in conclusion that the physical results of the limit transition $I \rightarrow \infty$ to the rigid superparamagnetic granular moment can be easily derived if in the Hamiltonian $H(\{\mu, \tau\})(3)$ we replace all the $\mu_{i}(n)$ by 1 . Then there appears a model of disordered magnet with generalized Ising superspins $N \tau_{i}$ which take two values: $+N$ and $-N$ and all the following calculations are the same as for the usual Ising magnet.

\section{CONCLUSION}

The granular magnets have a very important peculiarity - a sharp spatial inhomogenity of atomic structure and thus a sharp difference (in several orders) of intragranular and intergranular interactions in magnitude and in the spatial extension. Strong but short-ranged exchange interactions are responsible for appearing on the granules of gigantic magnetic moments and their superparamagnetic behaviour at intermediate temperatures. In this work we propose a microscopic scheme of taking into consideration these short-ranged intragranular interactions which is based on the idea of a separate consideration of intragranular and intergranular molecular fields and the following introduction of two types of magnetic order - the local one (on granule) and the global one (on the whole sample). This scheme is tested on the simplest Ising model, and it let us show, in particular, such a phenomenon, as the transition of the system from the usual paramagnetic spin behaviour to the "soft" superparamagnetic behaviour, and also a start of formation of low-temperature global magnetic order from the "soft" granular supermoments.

At the same time it is obvious, that it is not enough to use the Ising model for describing the important experimental properties. It is known that at experiments superparamagnetic behaviour is observed in systems of magnetic particles with only a narrow range of particle sizes [11]. On the one hand, the particle size should be greater than some critical size for us to be able to speak about the appearance of a spontaneous magnetic order in it. The experiments show (see, for example, [11]) that in isolated ferromagnetic particles with the size smaller than the critical one there is no magnetic ordering. On the other hand, the particle size has an upper limit because of the blocking effect which means that the relaxation time of sufficiently large particles (the time when magnetic moment of the granule as a whole reaches the thermal equilibrium state) can be larger than the time of experimental investigations. This phenomenon is connected with the effect of increasing the energy barrier (getting over by granular magnetic moment at it rotation) at increase of particle size. Besides the energy barrier is defined mainly with intrinsic anisotropy of the particle and, evidently, to a lesser degree with the weak intergranular exchange [11]. That is why it is clear, that description of blocking effects in granular magnets require the transition to vector magnetic models with taking into account the effects of final magnetic intragranular anisotropy.
[1] M. V. Medvedev, Phys. Met. Metallogr. 88, No 1, 9 (1999).

[2] E. E. Kokorina, M. V. Medvedev, Phys. Met. Metallogr. 89, No 6, 27 (2000).

[3] V. V. Kokorin, A. E. Perekos, Pis'ma Zh. Eksp. Teor. Fiz. 27, No 9, 500 (1978).

[4] D. Sherrington, S. Kirkpatrick, Phys. Rev. Lett. 35, No 261792 (1975).

[5] M. Kac, in: Ustojchivost' i fazovyje pieriekhody (F. Dyson, E. Montroll, M. Kac, M. Fisher, eds.) (Mir, Moscow, 1973). [Translated from English: (Gordon and Breach Science Publishers, New York, 1968)]
[6] K. H. Fisher, J. A. Hertz, Spin glasses, (Eds. Cambridge: Cambridge University Press, 1991).

[7] J. R. L. de Almeida, D. J. Thouless, J. Phys. A 11, No 5, 983 (1978).

[8] V. V. Kokorin, I. A. Osipenko, Phys. Met. Metallogr. (USSR) 54, 826 (1982).

[9] S. Katsura, Prog. Theor. Phys. 55, 1049 (1976).

[10] M. Suzuki, Prog. Theor. Phys. 58, 1151 (1977).

[11] I. S. Jacobs and C. P. Bean, Magnetism, edited by G. T. Rado, H. Suhl, (Academic Press, New York, London, 1963), p. 271. 


\title{
“М'ЯКИЙ" СУПЕРПАРАМАГНЕТНИЙ СТАН І МЕЖІ ЙОГО СТІӤКОСТИ В МОДЕЛІ ІЗИНГА НЕВПОРЯДКОВАНОГО ГРАНУЛЬОВАНОГО МАГНЕТУ
}

\author{
Е. Е. Кокоріна, М. В. Медведєв \\ Інститут електрофізики, Уральсъке відділення, Російсъка академія наук, \\ вул. Амундсена, 106, Екатеринбург, 620016, Росіл \\ E-mail: medvedev@ief.uran.ru
}

\begin{abstract}
Запропоновано модель Ізинга невпорядкованого гранульованого магнету. У пій моделі ідентичні гранули з приблизно $N \approx 10^{4}-10^{5}$ магнетними атомами хаотично поміщені в немагнетну матрицю, ізингівські спіни всередині кожної гранули взаємодіють між собою за допомогою сильної феромагнетної обмінної взаємодії, а спіни з різних гранул взаємодіють між собою за допомогою слабкої хаотичної обмінної взаємодії, яка описується несиметричним розподілом Гауса.

У пій моделі $\epsilon$,дві критичні температури магнетного впорядкування: спершу з'являється локальне феромагнетне впорядкування всередині гранул за відсутности далекосяжних кореляцій між спонтанними магнетними моментами різних гранул, потім при нижчій температурі з'являється далекосяжний суперферомагнетний стан або стан макроспінового скла у всьому зразку. Вище ві, верхньої критичної температури спостерігається звичайна парамагнетна поведінка ізингівських спінів, “м'який” суперпарамагнетний стан (iз залежними від температури абсолютними значеннями спонтанних магнетних моментів гранул) виявлено між верхньою та нижньою критичними температурами.

Функцію розподілу моделі розраховано в наближенні молекулярного поля, проаналізовано температурну залежність лінійної та нелінійної магнетної сприйнятливости в “м'якому" суперпарамагнетному стані. Також розраховано лінію нестійкости де Алмеіди-Таулеса при скінченних магнетних полях для випадку, коли в системі проявляється перехід від суперпарамагнетного стану в низькотемпературний стан макроспінового скла.
\end{abstract}

